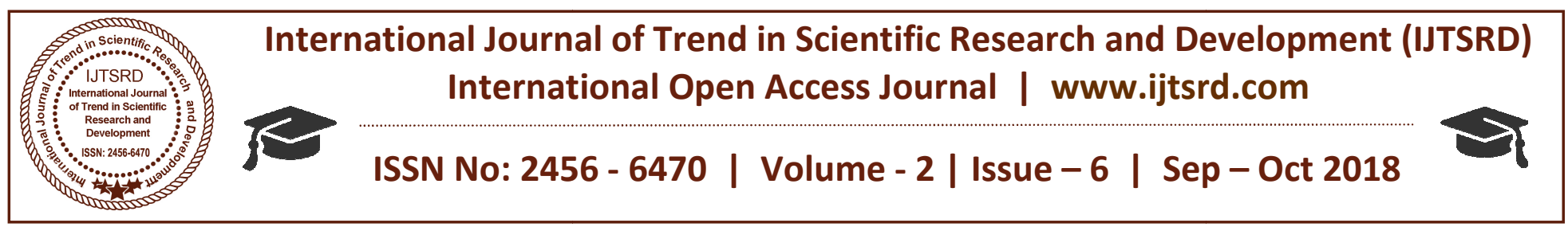

\title{
A Survey on Clustered and Energy Efficient Routing Protocols for Wireless Sensor Networks
}

\author{
Diksha Pandita $^{1}$, Ravi Kumar Malik ${ }^{2}$ \\ ${ }^{1}$ Research Scholar, ${ }^{2}$ Assistant Professor \\ Department of ECE, Geeta Engineering College, \\ Panipat Kurukshetra University, Kurukshetra, Haryana, India
}

\begin{abstract}
The main issue in WSN is energy limited characteristic of the sensor node. So the problem is to have the routing protocol in such the manner that it should be energy efficient in order to increase the life time of the whole WSN. Hierarchical routing architecture divides the whole network in to a group of cluster and only cluster head is responsible to forwarding the data to base station directly or via other cluster heads. In location based architecture of routing, localization of the sensor node is to be used to compute the path to the sensed data. During the creation of network topology, the process of setting up routes in WSNs is usually influenced by energy considerations, because the power consumption of a wireless link is proportional to square or even higher order of the distance between the sender and the receiver. Hierarchical routing can be centralized or non- centralized. In non-centralized hierarchical routing, the sensor nodes self-configures for the cluster head on the basis of selecting a random number. They don't consider the case of residual energy. But in centralized routing the base station is responsible to create cluster. In hierarchical routing architecture, sensor nodes self-configure themselves for the formation of cluster heads. In this paper, we have studied different types of hierarchical routing protocols for wireless sensor networks.
\end{abstract}

Keywords: WSN, hierarchical routing, SEP, TEEN, Issues in WSN, Applications of WSN

\section{INTRODUCTION}

The scattered nature and active topology of wireless sensor network (WSN) have some particular requirements that are as follows - the reduced energy consumption of the network, and maximizing the

network lifetime. These requirements should be met in routing protocols of wireless sensor network. Wireless sensor network consist of tiny nodes called sensor nodes. These nodes are battery powered and have limited energy for use. Also these nodes are randomly deployed over an area to monitor the happenings in the environment such as humidity, seismic events, temperature, pollution, fire detection etc [1]. These nodes are mobile or static depending on the physical phenomenon monitored. There are two types of WSN- unstructured and structured WSN [2]. Unstructured WSN - it is the one containing a large collection of tiny sensor nodes. The sensor nodes are deployed randomly in the area. The network is left unattended to perform monitoring and reporting functions. Whereas in structured WSN, all the nodes are deployed in a pre-planned manner. Network maintenance (managing connectivity and detecting failures) is quite difficult in unstructured WSN than structured WSN. Also, in structured WSN, the sensor nodes are placed at specific positions which help in providing full coverage, whereas there are uncovered areas in unstructured WSN.

The primary function of a sensor node is to collects data and sent it to the base station. A sensor node contains three basic components [3]:

A. Sensing Subsystem: - It is used for acquisition of data from the physical environment where nodes are deployed.

B. Processing Subsystem: - It is used for storing the data and local data processing is carried out.

C. Wireless Communication Subsystem: - It is used for data transmission. 
Also a power source consisting of non rechargeable batteries supplies the energy needed to perform the programmed task. As it is inconvenient to recharge the batteries because nodes when once deployed are unreachable to users. In some cases it is possible to scavenge the energy from the external environment, energy buffers are sometimes needed as well. But in any case, energy resource is very vital resource and must be used carefully. Therefore, energy conservation is the key issue in the design of systems based on wireless sensor network. To prolong the lifetime of network different techniques are applied. For example, energy efficient protocols are aimed for minimizing the energy consumption during network activities.

\section{HIERARCHICAL PROTOCOLS}

It is also known as Cluster Based Routing. The routing protocols in this category force a structure on the wireless sensor networks to provide energy efficient communication and scalability to the network. The network nodes are organized into clusters and based on some criteria a cluster head for each cluster is selected [5] i.e. higher energy nodes are used to route the data, and lower energy nodes are used to sense the area. Figure 1 illustrates the clustering based scheme of the wireless sensor network. The cluster heads are then responsible for organizing the activities within the cluster. Here, in hierarchical routing the number of messages that are transferred to the base-station are decreased due to fusion and data aggregation thus it lowers the energy consumption and hence helps to improve the lifetime of the network [5]. In such protocols the nodes around the base station and cluster head will exhaust their energy faster than all the other nodes.

Some of the well known protocols under this section are: -

A. Low Energy Adaptive Clustering Hierarchy (LEACH): - LEACH is a clustering based algorithm. Key features of LEACH are [7]: - First, to reduce the global communication it uses local compression. Second, it uses randomized rotation for making clusters. Third, for cluster setup and other operations localized coordination and control is required. Unlike, the conventional clustering algorithm, LEACH is self-organizing, adaptive clustering algorithm [8]. of the network. The non cluster head nodes have to decide to which cluster it wants to belongs by choosing the cluster heads which require less energy for communication. Once the nodes are organized into clusters each cluster head creates a schedule for all the nodes belonging to its cluster.

B. Low Energy Adaptive Clustering Hierarchy Centralized (Leach-C): -Since LEACH has a problem of determining the number of cluster heads in every round. For the formation of clusters, LEACH-C utilizes the base-station [6]. In LEACH the nodes configure themselves to form clusters, but in LEACH-C at each iteration or round the base-station first receives the information about location, state and the energy level of the nodes. This information is used then to calculate the mean value of network node energy; and base-station finds predetermined number of cluster heads and hence configures the network into clusters [6]. The nodes that are selected as cluster head have higher energy level that the mean energy value. LEACH-C is an improvement over LEACH by the following points: First, to produce clusters that require less energy for transmission Base-Station utilizes it global knowledge of the network, Second, unlike LEACH where the number of cluster head varies from round to round due to lack of global coordination amongst nodes, in LEACH-C the number of cluster heads in each round equals a predetermined optimal value [7].

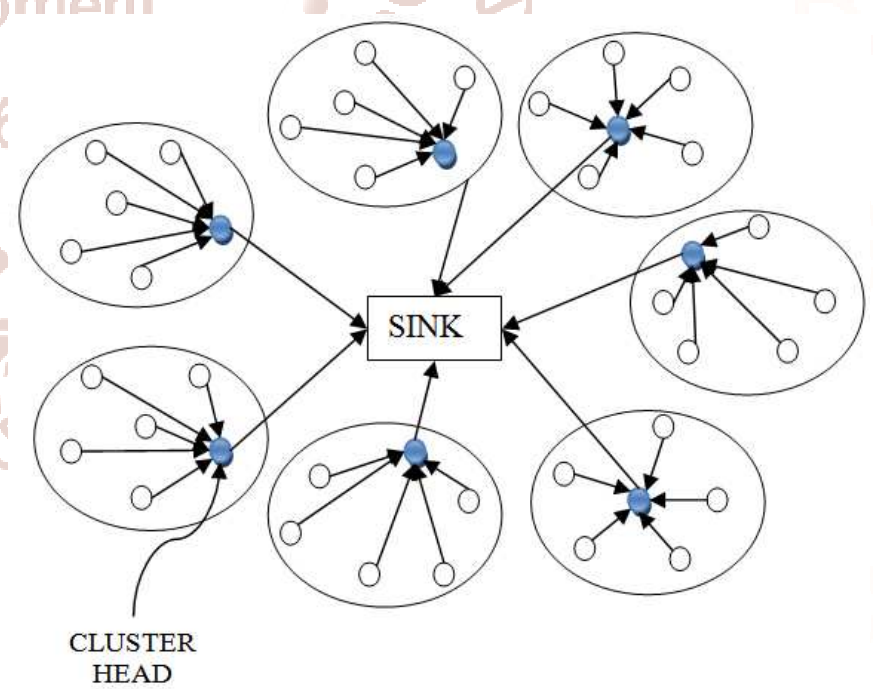

Figure 1: Clustering Schema for Wireless Sensor Network (redrawn from [6]).

\section{Stable Election Protocol (SEP):}

A percentage of the population of sensor nodes is equipped with more energy resources than the rest of the nodes. Let $\mathrm{m}$ be the fraction of the total number of nodes $\mathrm{n}$, which are equipped with $\alpha$ times more energy than the others. We refer to these powerful 
nodes as advanced nodes, and the rest $(1-\mathrm{m}) \times \mathrm{n}$ as normal nodes. We assume that all nodes are distributed randomly over the sensor field [7].

Suppose that Eo is the initial energy of each normal sensor. The energy of each advanced node is then Eo* $(1+\alpha)$. The total (initial) energy of the new heterogeneous setting is equal to:

$$
\mathrm{n} * \text { Eo } *(1+\alpha * \mathrm{~m})
$$

So, the total energy of the system is increased by a factor of $1+\alpha * \mathrm{~m}$.

A. each normal node becomes a cluster head once every 1 popt $\cdot(1+\alpha \cdot \mathrm{m})$ rounds per epoch;

B. each advanced node becomes a cluster head exactly $1+\alpha$ times every 1 popt $\cdot(1+\alpha \cdot \mathrm{m})$ rounds per epoch;

C. the average number of cluster heads per round per epoch is equal to $n \times$ popt

Cluster Head Election for normal nodes is based on following equation:

$$
T\left(s_{n r m}\right)= \begin{cases}\frac{P_{n r m}}{1-p_{n i m} \cdot\left(r \bmod \frac{1}{p n r m}\right)} & \text { if } s_{n r m} \in G^{\prime} \\ 0 & \text { otherwise }\end{cases}
$$

where $r$ is the current round, $\mathrm{G}^{\prime}$ is the set of normal nodes that have not become cluster heads within the last $1 /$ pnrm rounds of the epoch, and $\mathrm{T}(\mathrm{snrm})$ is the threshold applied to a population of $\mathrm{n} \cdot(1-\mathrm{m})$ (normal) nodes. This guarantees that each normal node will become a cluster head exactly once every 1/popt- $(1+\alpha \cdot \mathrm{m})$ rounds per epoch, and that the average number of cluster heads that are normal nodes per round per epoch is equal to $n \cdot(1-\mathrm{m}) \times$ pnrm.

Cluster Head Election for advanced nodes is based on following equation:

$$
T\left(s_{a d v}\right)= \begin{cases}\frac{p_{a d v}}{1-p_{a d v} \cdot\left(r \bmod \frac{1}{p_{a d v}}\right)} & \text { if } s_{a d v} \in G^{\prime \prime} \\ 0 & \text { otherwise }\end{cases}
$$

where $G^{\prime \prime}$ is the set of advanced nodes that have not become cluster heads within the last 1 padv rounds of the epoch, and $\mathrm{T}(\mathrm{sadv})$ is the threshold applied to a population of $\mathrm{n} \quad \mathrm{x} \quad \mathrm{m}$ (advanced) nodes. This guarantees that each advanced node will become a cluster head exactly once every $(1 /$ popt $) \times((1+\alpha \cdot \mathrm{m}) /(1+\alpha))$ rounds.

\section{Threshold sensitive Energy Efficient sensor Network protocol (TEEN):}

In this section, we present a new network protocol called TEEN. It is targeted at reactive networks and is the first protocol developed for reactive networks. In this scheme, at every cluster change time, in addition to the attributes, the cluster-head broadcasts to its members, Hard Threshold (HT): This is a threshold value for the sensed attribute. It is the absolute value of the attribute beyond which, the node sensing this value must switch on its transmitter and report to its cluster head. Soft Threshold (ST): This is a small change in the value of the sensed attribute which triggers the node to switch on its transmitter and transmit.

The nodes sense their environment continuously. The first time a parameter from the attribute set reaches its hard threshold value, the node switches on its transmitter and sends the sensed data. The sensed value is stored in an internal variable in the node, called the sensed value (SV). The nodes will next transmit data in the current cluster period, only when both the following conditions are true:

1. The current value of the sensed attribute is greater than the hard threshold.

2. The current value of the sensed attribute differs from SV by an amount equal to or greater than the soft threshold. Whenever a node transmits data; $\mathrm{SV}$ is set equal to the current value of the sensed attribute.

Thus, the hard threshold tries to reduce the number of transmissions by allowing the nodes to transmit only when the sensed attribute is in the range of interest. The soft threshold further reduces the number of transmissions by eliminating all the transmissions which might have otherwise occurred when there is little or no change in the sensed attribute once the hard threshold.

The main features of this scheme are as follows:

1. Time critical data reaches the user almost instantaneously.

2. So, this scheme is eminently suited for time critical sensing applications.

3. Message transmission consumes much more energy than data sensing. So, even though the nodes sense continuously, the energy consumption in this scheme can potentially be much less than in the proactive network, because data transmission is done less frequently.

4. The soft threshold can be varied, depending on the criticality of the sensed attribute and the target application. 
5. A smaller value of the soft threshold gives a more accurate picture of the network, at the expense of increased energy consumption. Thus user can control the trade-off between energy efficiency and accuracy.

6. At every cluster change time, the attributes are broadcast a fresh and so, the user can change them as required.

The main drawback of this scheme is that, if the thresholds are not reached, the nodes will never communicate; the user will not get any data from the network at all and will not come to know even if all the nodes die. Thus, this scheme is not well suited for applications where the user needs to get data on a regular basis. Another possible problem with this scheme is that a practical implementation would have to ensure that there are no collisions in the cluster.

TDMA scheduling of the nodes can be used to avoid this problem. This will however introduce a delay in the reporting of the time-critical data. CDMA is another possible solution to this problem.

\section{APPLICATIONS OF WSN}

Wireless sensor networks are used in variety of everyday life activities or services. The most common application of WSN is for monitoring where we randomly deploy some nodes to monitor some phenomenon. We categorize the applications of WSN in military, environment, health home and other commercial areas.

A. Military Applications:- Wireless sensor networks can be an integral part of military Command, Control, Communication, Computing, Intelligence, Surveillance, Reconnaissance and Targeting [6]. The rapid deployment, selforganization and fault tolerance characteristics of sensor networks make them a very promising sensing technique for military. The military applications of sensor networks are monitoring friendly forces, biological and chemical attack detection, targeting, battle damage assessment etc.

B. Environmental Applications: - The environmental applications of WSN are tracking the movement of birds, monitoring the environmental conditions that effect crops and livestock's, precision agriculture, pollution study, forest fire detection, meteorological or geophysical research, biocomplexity mapping of the environment [3].

C. Health Applications: - This area of WSN provide interfaces for the disabled, integrated patient monitoring, tracking and monitoring doctors and patients inside the hospital, it also offer significant cost saving and enable new functionalities that will assist people with chronic disease on daily activities etc [5].

D. Home Applications: -Home applications include automation and smart environment. In home automation the sensors are buried in the domestic devices that will help them to interact with each other and with the external environment via internet. And, it will allow user to coordinate these devices locally and remotely [3].

\section{CRITICAL ISSUES OF WIRELESS SENSOR NETWORKS}

The main design goal of WSN is to not only transmit the data between source and destination but is also to increase the network lifetime; which can achieved by applying energy efficient protocols. The task of the protocol is not only to choose a path having lower energy consumption between sources to destination (BS), but also to find an efficient approach to prolong the network lifetime. But sometimes continuous use of low energy path may lead to energy depletion of sensor nodes in that path leading to network partition.

Performance of the routing protocol is calculated based on some terms which includes [1]:-

A. Energy per Packet: - the amount of energy required sending a packet from source to destination safely.

B. Low Energy Consumption: - it includes such protocols that considers the remaining energy level of nodes and selects route accordingly for longevity of the network.

C. Total Number of Nodes Alive: - It gives an idea of the area coverage of the network over time. This metrics is related to network lifetime.

D. Average Packet Delay: - This metrics measure the accuracy of packet. This metrics is calculated as one way latency which is observed between transmission and reception of data packet at the sink.

E. Time until the First Node Dies: - This metric indicates the duration for which all the sensor nodes on the network are alive. There are protocols in which the first node on the network runs out of energy earlier than in other protocols, but manages to keep the network operational much longer.

F. Energy Spent Per Round: - This metric is related to the total amount of energy spent in routing messages in a round. It is a short-term measure 
designed to provide an idea of the energy efficiency of any proposed method in a particular round.

G. Packet Size: - the lifetime of the network also depends on the packet size; it determines the time that a transmission will last. As it is effective in energy consumption so packet size should be reduced by combining large number of packets.

H. Distance: - The distance between the transmitter and receiver can affect the power that is required to send and receive packets. The routing protocols can select the shortest paths between nodes and reduce energy consumption.

\section{CONCLUSION}

WSNs differ from traditional wireless communication networks in several of their characteristics. One of them is power awareness, due to the fact that the batteries of sensor nodes have a restricted lifetime and are difficult to be replaced. Therefore, all protocols must be designed in such a way as to minimize energy consumption and preserve the longevity of the network. That is why, routing protocols in WSNs aim mainly to accomplish power conservation while in traditional networks they focus primarily on the Quality of Service (QoS). In this paper, we have surveyed hierarchical routing protocols in wireless sensor network. From the above study we can conclude that there are many routing protocols and you can use any one of them for your network based on the application. All these protocols use static sink or BS for data transmission which leads to energy hole problem. In future, we will propose mobile-sink and multi-sink strategies to remove energy hole problem. And, it may enhance packet delivery and network lifetime.

\section{REFERENCES}

1. Nikolaos A. Pantazis, Stefanos A. Nikolidakis, Dimitrios D. Vergados, "Energy-Efficient Routing Protocols in Wireless Sensor Networks: A Survey," IEEE Communications Surveys \& Tutorials, 2013, Vol. 15, Issue. 2, pp. 551-590.

2. Yick J., Mukherjee B., Ghosal D., "Wireless Sensor Network Survey," Computer networks, 2008, Vol. 52, Issue. 12, pp. $2292-2330$. 